\title{
Preradiotherapy MR Imaging: A Prospective Pilot Study of the Usefulness of Performing an MR Examination Shortly before Radiation Therapy in Patients with Glioblastoma
}

\author{
(DC. Majós, (DM. Cos, (D). Castañer, (iD) A. Pons, (D). Gil, (D)A. Fernández-Coello, (DM. Macià, (D). Bruna, and (D). Aguilera
}

\begin{abstract}
BACKGROUND AND PURPOSE: Current protocols in patients with glioblastoma include performing an MR examination shortly after surgery and then 2-6 weeks after ending concomitant chemoradiotherapy. The assessment of this first postradiotherapy examination is challenging because the pseudoprogression phenomenon may appear. The aim of this study was to explore if performing an MR examination shortly before radiation therapy (preradiotherapy MR imaging) could improve the radiologic assessment of patients with glioblastoma.
\end{abstract}

MATERIALS AND METHODS: A preradiotherapy MR imaging examination was prospectively performed before the start of radiation therapy in 28 consecutive patients with glioblastoma who had undergone surgical resection. Tumor response to chemoradiotherapy was assessed twice: with the early postoperative MR examination as baseline and with the preradiotherapy MR imaging examination as baseline. In addition, tumor growth in the preradiotherapy MR imaging examination was evaluated, and its correlation with patient survival was assessed with Kaplan-Meier analysis and Cox regression.

RESULTS: Tumor progression after radiation therapy was found in 16 patients, corresponding to pseudoprogression in 7 of them (44\%). Four assessments of pseudoprogression switched to partial response or stable disease when preradiotherapy MR imaging was the baseline examination, and the ratio of pseudoprogression was reduced to $25 \%$ ( 3 of 12). Significant differences in survival were found when patients were stratified according to the pattern of tumor growth on preradiotherapy MR imaging (median overall survival "no-growth," 837 days; "focal-growth," 582 days; "global-growth," 344 days; $P=.001$ ).

CONCLUSIONS: Performing a preradiotherapy MR imaging examination may improve the clinical management of patients with glioblastoma by reducing the ratio of pseudoprogression assessments and providing prognostic information.

ABBREVIATIONS: $E$ PMR = early postoperative MR imaging; PRMR = preradiotherapy MR imaging

G lioblastoma is the most common and aggressive type of glioma. ${ }^{1}$ The current standard of care for patients newly diagnosed with glioblastoma involves maximum safe surgical resec-

Received April 15, 2016; accepted after revision July 1.

From the Departments of Radiology, Institut de Diagnòstic per la Imatge (C.M., M.C., S.C., A.P., C.A.), Medical Oncology, Institut Català d'Oncologia L'Hospitalet (M.G.), Neurosurgery (A.F.-C.), Radiotherapy Oncology, Institut Català d'Oncologia L'Hospitalet (M.M.), and Neurology (J.B.), Hospital Universitari de Bellvitge, L'Hospitalet de Llobregat, Spain; Centro de Investigación Red en Bioingeniería, Biomateriales y Nanomedicina (C.M., C.A.), Cerdanyola del Vallès, Spain; and Institut d'Investigació Biomèdica de Bellvitge, IDIBELL (J.B.), L'Hospitalet de Llobregat, Spain. This work was supported in part by a grant from the Grupo Español de Investigación en Neurooncología (GEINO) and by the Centro de Investigación Red en Bioingeniería, Biomateriales y Nanomedicina (CIBER-BBN), which is an initiative of the VI National R\&D\&i Plan 2008-2011, CIBER Actions and financed by the Instituto de Salud Carlos III with assistance from the European Regional Development Fund. Please address correspondence to Carles Majós, MD, Institut de Diagnòstic per la Imatge, Hospital Duran i Reynals, Gran Via de l'Hospitalet 199, 08908 L'Hospitalet de Llobregat, Barcelona, Spain; e-mail: cmajos@bellvitgehospital.cat

- Indicates open access to non-subscribers at www.ajnr.org

http://dx.doi.org/10.3174/ajnr.A4917 tion followed by radiation therapy plus concomitant and adjuvant temozolamide. ${ }^{2,3}$ MR imaging plays a pivotal role in the follow-up of brain glioblastomas after treatment. Guidelines suggest performing an MR examination early after surgery to evaluate the extent of surgical resection, another examination 2-6 weeks after completion of concomitant treatment with radiation therapy and temozolamide to evaluate response, and then every $2-4$ months for follow-up. ${ }^{4,5}$ The examination performed 2-6 weeks after completion of radiation therapy represents a major challenge for multidisciplinary neuro-oncology units. This is because the pseudoprogression phenomenon may appear in this timeframe. It is well known that approximately half of the patients who show progressive disease on an MR examination performed up to 3 months after concomitant temozolamide-radiation therapy improve or stabilize on subsequent assessments without any change in therapy. This phenomenon is termed pseudoprogression. ${ }^{6}$ Therefore, 2 possibilities should be taken into account when an enhancing-lesion enlargement is found in the first postradiation 

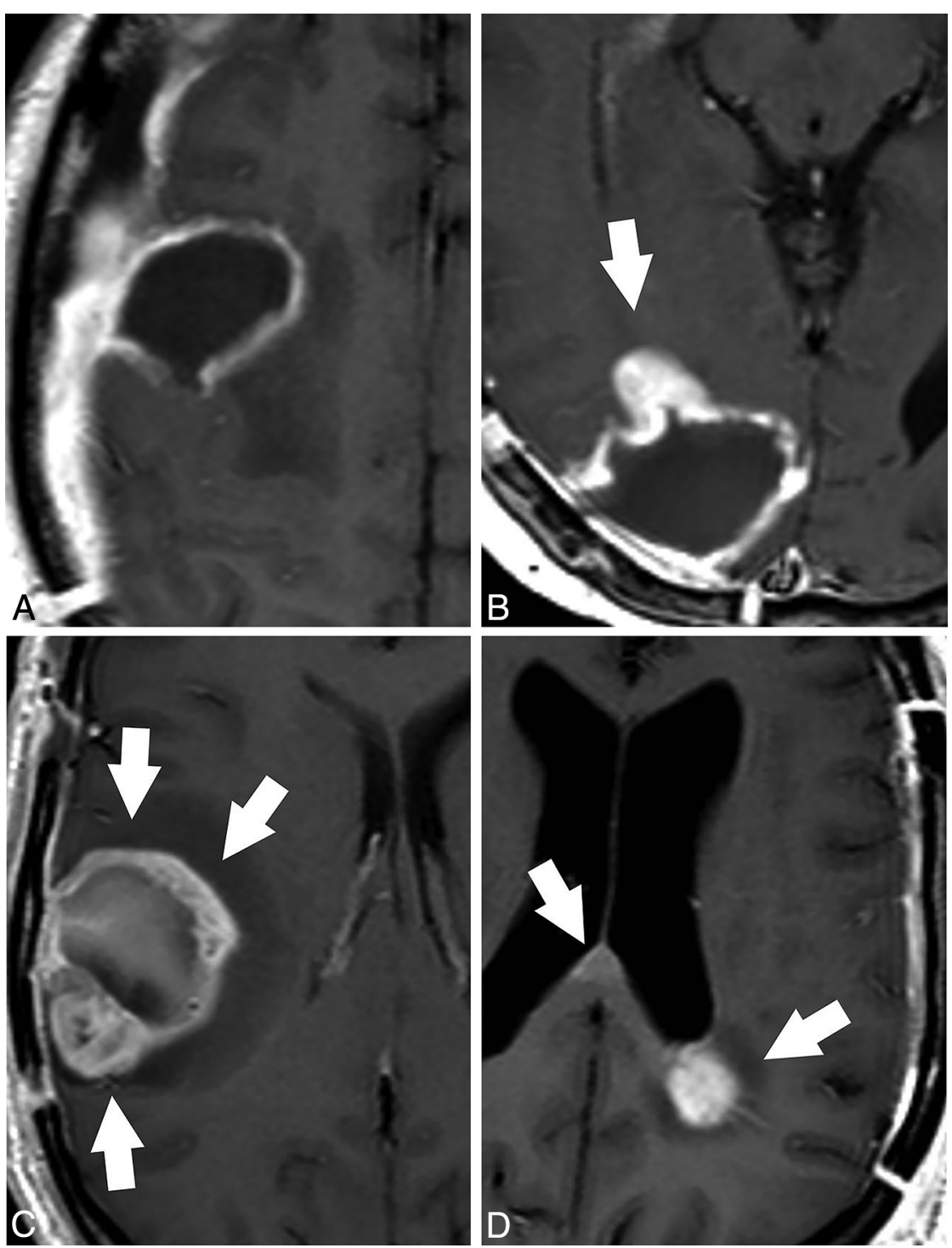

FIG 1. Patterns of tumor growth in the PRMR examination in patients with glioblastoma. Axial contrast-enhanced T1-weighted images. A, No growth. The MR examination shows a thin homogeneous enhancement in the wall of the surgical cavity that is considered normal evolution after surgery. B, Focal growth. A focal-enhancing nodule is found at the anterior margin of the surgical cavity in this study (arrow). C, Global growth. Thick irregular enhancement in the margins of the surgical cavity involving more than half of the surgical cavity (arrows). Note the presence of some hyperintense postsurgical material in the surgical cavity. D, Distant growth. Focal contrast enhancement is found in the juxtaventricular parietal lobe and in the splenium of the corpus callosum (arrows), distant to the surgical cavity (not shown), after resection of a left temporal lobe glioblastoma.

MR examination: true progression and pseudoprogression. Differentiation between the 2 entities has important clinical consequences. Patients with pseudoprogression will benefit from following adjuvant treatment with temozolamide, whereas an alternative treatment should be started as soon as possible in patients with true progression. Unfortunately, it is not currently possible to confidently discriminate between the 2 entities with MR imaging in this situation. ${ }^{7}$

Pseudoprogression has been related to vasodilation and disruption of the $\mathrm{BBB}$ caused by radiation toxicity, tumor necrosis caused by treatment effectiveness, and local tissue inflammatory reaction. ${ }^{6,8}$ An additional possibility is that tumor growth between surgery and the start of radiation therapy plays a role in this phenomenon. Radiation therapy should preferably be initiated into 6 weeks after surgery. Nevertheless, wound healing and the patient's clinical situation may require a delay in the start of treatment, and some recent studies have reported a time range up to 14 weeks from surgery to initiation of radiation therapy., ${ }^{9,10}$ Glioblastoma is a highly proliferative tumor that may show relevant growth in this period of time. ${ }^{9}$ However, the first postradiation examination is usually compared with the early postoperative MR imaging (EPMR) examination to assess tumor response, and tumor growth before starting treatment may be wrongly attributed to tumor progression.

Our intention in this study was to assess the impact of performing an MR examination shortly before the start of radiation therapy, hereafter referred to as the preradiotherapy $\mathrm{MR}$ imaging (PRMR) examination, in the management of patients with glioblastoma who have undergone surgical resection of the tumor. Our hypothesis was that tumor growth before radiation therapy could play a role in some cases classified as pseudoprogression. If this is true, a number of patients who show contrastenhancing lesion enlargement in the first postradiation MR examination compared with the EPMR examination, and who will be considered as experiencing pseudoprogression, could show stable disease or partial response when the examination is compared with the PRMR examination. Accordingly, the prevalence of pseudoprogression should decrease when PRMR is used as the baseline examination. Another aspect that we wanted to explore was whether the evolution of the tumor between EPMR and PRMR could provide an assessment of the proliferative behavior of the tumor and if it could be of value in predicting the outcome after therapy. To do this, we evaluated the correlation between the pattern of tumor growth on the PRMR examination and patient overall survival.

\section{MATERIALS AND METHODS Patient Selection}

This prospective study was approved by the ethics committee of Hospital Universitari de Bellvitge, and informed consent was obtained from all patients. The inclusion criteria for this study were as follows: surgical resection of a newly diagnosed tumor; histopathologic diagnosis of glioblastoma on the basis of the World 
Health Organization criteria; EPMR available, including T1weighted imaging, contrast-enhanced T1-weighted imaging, FLAIR imaging, and DWI; scheduled treatment with concomitant radiation therapy and temozolamide; and informed consent of the patient. Thirty consecutive patients studied in our hospital fulfilled the inclusion criteria and were prospectively included in the study between December 2012 and October 2014. Two patients showed quick clinical deterioration after surgery and did not start treatment with radiation therapy. These 2 patients were excluded from the study. The remaining 28 patients constituted the main body of the study.

\section{MR Imaging}

All patients included in the study underwent MR imaging within 7 days before the start of radiation therapy. Follow-up MR imaging was performed 2-6 weeks after completion of radiation therapy in all patients and then every 2-4 months until the end of the study in February 2016.

All MR examinations were performed in a $1.5 \mathrm{~T}$ MR unit (Achieva; Philips Healthcare, Best, the Netherlands) and included, at least, T1-weighted imaging, contrast-enhanced T1weighted imaging, FLAIR imaging, and DWI sequences. T1weighted images were acquired with TR, $500 \mathrm{~ms}$; TE, $12 \mathrm{~ms}$; FOV, $23 \mathrm{~cm}$; acquisition matrix, $256 \times 192$ pixels; and section thickness, $5 \mathrm{~mm}$ with a $1-\mathrm{mm}$ gap. FLAIR MR images were obtained

Table 1: Clinical characteristics of the patients

\begin{tabular}{lc}
\hline \multicolumn{1}{c}{ Characteristics } \\
\hline Age (yr) (mean [range]) & $57[26-73]$ \\
Sex (No. of patients) & 19 \\
$\quad$ Men & 9 \\
Women & 9 \\
Karnofsky Performance Status (No. of patients) & 19 \\
$\quad 70-80$ & \\
$90-100$ & 2 \\
Time between surgery and EPMR (No. of patients) & 15 \\
$\quad<24 \mathrm{~h}$ & 11 \\
24-48 h & $39[27-64]$ \\
$48-72 \mathrm{~h}$ & \\
Time between surgery and radiotherapy & \\
$\quad$ (d) (mean [range]) & \\
Time between PRMR and radiotherapy & 8 \\
$\quad$ (No. of patients) & 9 \\
1d & 2 \\
2-3 d & $28[16-39]$ \\
$4-5 \mathrm{~d}$ & \\
6-7 d & \\
Time between radiotherapy and follow-up & \\
$\quad$ with MRI (d) (mean [range]) &
\end{tabular}

with TR, 11,000 ms; TE, 140 ms; TI, 2800 ms; FOV, 23 cm; acquisition matrix, $256 \times 192$ pixels; and section thickness, $5 \mathrm{~mm}$ with a 1-mm gap.

Three neuroradiologists (C.M., M.C., and S.C.) with 20, 12, and 13 years of experience in neuro-oncology MR imaging, respectively, blinded to survival data independently evaluated the MR examinations at 2 different times: in the early postoperative period (EPMR) and in the early preradiotherapy period (PRMR). Disagreements between readers were resolved by consensus.

The EPMR was assessed according to previously established criteria. ${ }^{11,12}$ Patients were stratified into 3 groups according to the extent of tumor resection: total resection, subtotal resection between 95\%-100\%, and subtotal resection $<95 \%$.

Tumor growth in the PRMR was assessed by evaluating the enhancing component of the tumor. Four possibilities were envisaged: no growth, focal growth, global growth, and distant growth. Appearance of a thin homogeneous enhancement in the wall of the surgical cavity was considered to be the normal evolution after surgery, and "no growth" was considered. Abnormal growth of the enhancing component was considered focal when it involved less than half of the postsurgical cavity and global when it involved more than half of the wall. When new contrast-enhancement foci were found without contact with the surgical cavity, distant growth was considered (Fig 1). The findings of the PRMR examination were reported to the oncologist to evaluate the need for any change in the field of radiation therapy. Changes in treatment planning were reported.

Response to the treatment was assessed according to the Response Assessment in Neuro-Oncology criteria by comparing the first postradiation MR examination to the EPMR examination. Four possibilities were envisaged: complete response, partial response, stable disease, and progressive disease. ${ }^{7}$ When progressive disease was found, we differentiated true progression from pseudoprogression by evaluating an MR examination performed more than 3 months after radiation therapy. If tumor growth was found in this examination, then the response was considered true progression. If the enhancing lesion was stable or decreased in size, then a diagnosis of pseudoprogression was made retrospectively. The same procedure was performed a second time using PRMR as the baseline examination, and the response was reassessed by comparing the first postradiation MR examination to the PRMR examination. Differences between the 2 assessments were reported.

\section{Statistical Analysis}

Patient overall survival was defined as the time from surgery until

Table 2: Concordance among tumor response assessment performed 2-6 weeks after completion of radiotherapy with both EPMR and PRMR as baseline exams ${ }^{a}$

\begin{tabular}{|c|c|c|c|c|c|}
\hline \multirow[b]{2}{*}{ PRMR as Baseline } & \multicolumn{5}{|c|}{ EPMR as Baseline } \\
\hline & $\begin{array}{c}\text { True } \\
\text { Progression }^{\mathrm{b}} \\
\end{array}$ & Pseudoprogression $^{\mathrm{b}}$ & $\begin{array}{c}\text { Stable } \\
\text { Disease }\end{array}$ & $\begin{array}{c}\text { Partial } \\
\text { Response }\end{array}$ & Total \\
\hline True Progression $^{\mathrm{b}}$ & 9 & 0 & 0 & 0 & 9 \\
\hline Pseudoprogression $^{\mathrm{b}}$ & 0 & 3 & 0 & 0 & 3 \\
\hline Stable Disease & 0 & 3 & 8 & 1 & 12 \\
\hline Partial Response & 0 & 1 & 1 & 2 & 4 \\
\hline Total & 9 & 7 & 9 & 3 & 28 \\
\hline
\end{tabular}

\footnotetext{
a Patients: $n=28$.
}

${ }^{b}$ Discrimination between true progression and pseudoprogression in patients showing progressive disease was performed by evaluating an MR exam performed more than 3 months after radiotherapy. death. For those patients who survived past the date of our analysis, survival was right censored in the survival analysis to the time the patient was last known to be alive. Two parameters were correlated to overall survival: the extent of the surgical resection evaluated in the EPMR examination and the pattern of tumor growth in the PRMR examination. Survival curves were constructed with the Kaplan-Meier method, and differences were evaluated with the log-rank 

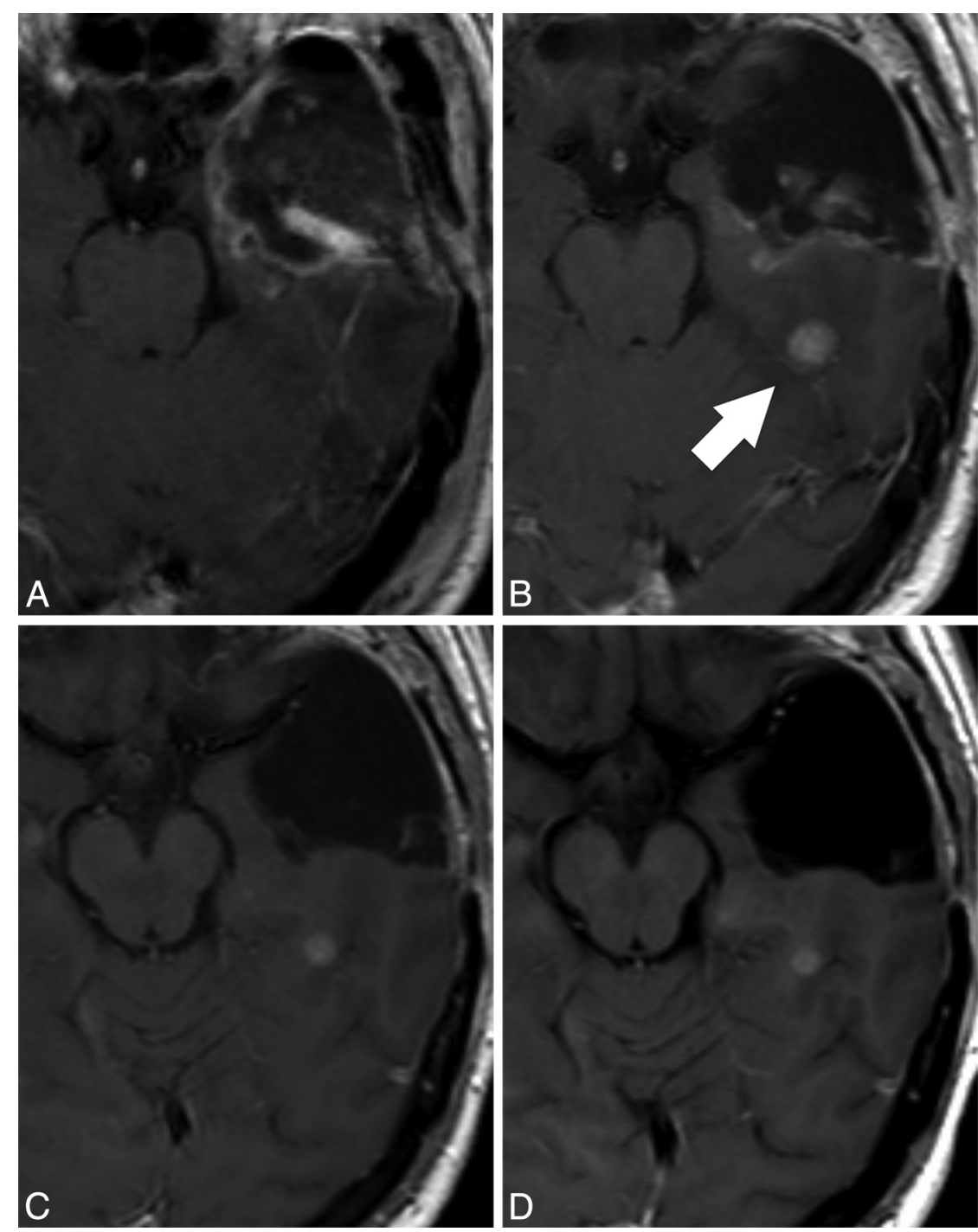

FIG 2. Representative case in which the response assessment is progressive disease (pseudoprogression) in reference to the EPMR examination and stable disease in reference to the PRMR examination. Contrast-enhanced Tl-weighted images performed at $A, 24$ hours after surgery (EPMR), $B, 3$ days before radiation therapy (PRMR), C, 27 days after radiation therapy, and $D, 135$ days after radiation therapy. A contrast-enhancing nodule appears in the PRMR (arrow, B), distant to the surgical cavity. As a relevant consequence, the radiation therapy field design was modified to include the nodule in the target volume. The postradiation examination $(C)$ shows a small decrease in the size of the nodule, which remains stable in an MR examination performed 4 months after radiation therapy $(D)$. In this particular case, the PRMR provides evidence that the nodule appeared before treatment and remained stable after the treatment. The response assessment would be progressive disease compared with the EPMR (pseudoprogression because the nodule remained stable in the next follow-up MR examination performed 4 months after radiation therapy) and stable disease compared with the PRMR.

test. Multivariable analysis with the variables "extent of tumor resection" and "pattern of contrast enhancement growth" was performed by fitting the Cox proportional hazards models to test a possible dependence between both variables, and the hazard ratio for death was estimated for each variable. Finally, we tested differences in the time elapsed between surgery and PRMR among patterns of growth with the Kruskal-Wallis test. All statistics were computed with PASW Statistics 18 software (IBM, Armonk, New York). Significant differences were determined by using a level of $P<.05$.

\section{RESULTS}

A summary of patient data and time elapsed between surgery, EPMR, PRMR, and radiation therapy can be found in Table 1. At the time of last assessment (February 2016), 8 patients were alive after 778 days of median follow-up (range, 637-945 days). Follow-up of deceased patients ranged between 78 and 1115 days (median, 444 days).

\section{Radiation Therapy Treatment Planning}

The field of radiation therapy planned with the EPMR examination had to be redesigned in 4 patients on the basis of the PRMR findings. The reason for this change was distant growth in 3 patients and large local growth in 1 additional patient. Survival of these patients was 213, 286, 334, and 1115 days.

\section{Tumor Response Assessment}

Tumor response to concomitant treatment with radiation therapy and temozolamide is shown in Table 2. The crosstable shows the correlation of the response assessment in the first postradiation MR examination when either the EPMR or the PRMR examinations were used as baseline. Sixteen patients showed progressive disease in the first postradiation examination when EPMR was used as baseline, corresponding to pseudoprogression in 7 patients and true progression in 9 (pseudoprogression ratio with EPMR as baseline examination, 7 of $16=44 \%$; positive predictive value for true progression when tumor growth is found on the first postradiation MR examination, 9 of $16=56 \%)$. Then, the response was reassessed with PRMR as baseline examination. Four patients who were classified as pseudoprogression with EPMR as baseline were classified as stable disease or partial response with PRMR as baseline. As a consequence, the number of patients who showed progressive disease was reduced to 12 when PRMR was used as baseline, corresponding to pseudoprogression in 3 patients and true progression in 9 patients (pseudoprogression ratio with PRMR as baseline, 3 of $12=25 \%$; positive predictive value for true progression, 9 of $12=75 \%$ ). Fig 2 shows a representative case in which tumor response was classified as pseudoprogression when the first postradiation MR examination was compared with the EPMR examination. In this case, PRMR showed that 
Table 3: Overall survival of patients stratified by the extent of resection evaluated on the EPMR and by the pattern of tumor growth on the PRMR

\begin{tabular}{|c|c|c|c|c|c|}
\hline \multirow[b]{2}{*}{ Characteristic } & \multicolumn{2}{|c|}{ Patients } & \multirow{2}{*}{$\begin{array}{c}\text { Survival (d) } \\
\text { (median; range) }\end{array}$} & \multirow{2}{*}{$\begin{array}{l}\text { Hazard Ratio } \\
(95 \% \mathrm{Cl})\end{array}$} & \multirow[b]{2}{*}{$P$ Value } \\
\hline & Total & Alive & & & \\
\hline $\begin{array}{l}\text { Extent of resection evaluated on } \\
\text { the EPMR }\end{array}$ & & & & & .002 \\
\hline Total & 7 & 4 & $835 ; 169-961$ & $0.099(0.022-0.445)$ & \\
\hline Between $95 \%$ and $100 \%$ & 11 & 4 & $637 ; 213-1115$ & $0.188(0.060-0.591)$ & \\
\hline Below $95 \%$ & 10 & 0 & $386 ; 78-577$ & $1^{\mathrm{b}}$ & \\
\hline Pattern of growth on the PRMR & & & & & .001 \\
\hline No growth & 7 & 4 & $837 ; 508-961$ & $0.047(0.006-0.393)$ & \\
\hline Focal & 10 & 3 & $582 ; 279-758$ & $0.294(0.098-0.885)$ & \\
\hline Global & 8 & 1 & $344 ; 78-835$ & $1^{\mathrm{b}}$ & \\
\hline Distant $^{a}$ & 3 & 0 & $334 ; 286-1115$ & NE & \\
\hline
\end{tabular}

Note:-NE indicates not evaluated

${ }^{\text {a }}$ Distant growth was not evaluated because of low number of cases.

b Used as reference category.

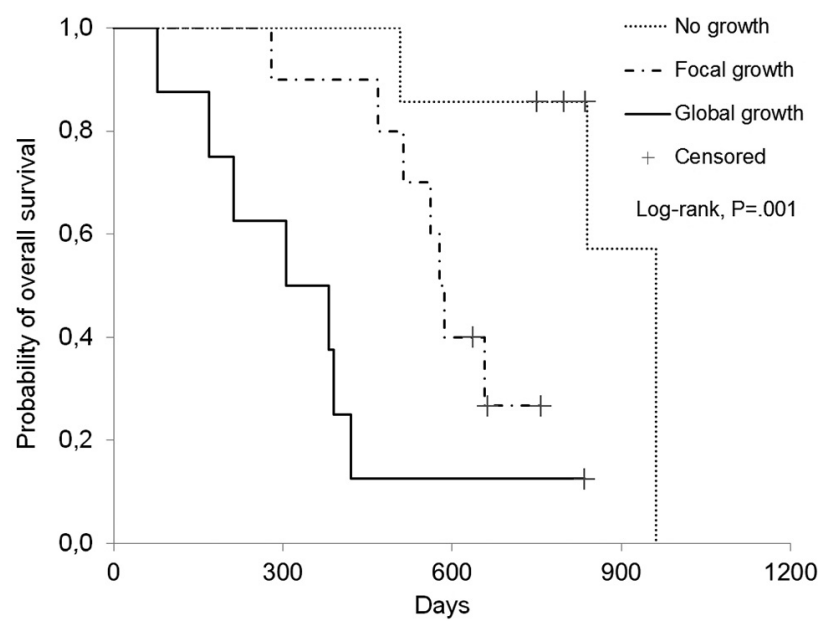

FIG 3. Survival curves of patients stratified according to the pattern of tumor growth in the PRMR. Patients with "no growth" in the PRMR showed significantly improved survival (median survival, 837 days) compared with patients with "focal growth" (median survival, 582 days) and "global growth" (median survival, 344 days; $P=.001$ ).

distant enhancement growth occurred before starting radiation therapy, and the response was changed to stable disease when the postradiation MR examination was compared with the PRMR examination.

\section{Tumor Growth Pattern in the PRMR}

Data about extent of tumor resection, tumor growth on PRMR, and patient survival are shown in Table 3. Significant differences in survival were found among patterns of enhancement on PRMR (Fig 3). The multivariable analysis showed these differences to be independent of the extent of tumor resection. The most favorable situation for the patients was to show a "no growth" pattern (7 patients; 4 alive; hazard ratio $=0.047)$. The next favorable situation was to show a "focal growth" pattern (10 patients; 3 alive; hazard ratio $=0.294)$. Showing a "global growth" pattern was the least favorable situation (8 patients; 1 alive; used as a reference to calculate hazard ratio). Growth distant to the enhancements seen on the EPMR examination was found in 3 patients on the PRMR examination (all deceased after 286, 334, and 1115 days, respectively). We considered that a statistical analysis of survival in these 3 patients could not be performed because of the low number of cases. Nevertheless, we would like to note that 1) the median survival of patients in this group was similar to "global growth";2) the longest surviving patient was included in this group; and 3) the volume targeted for radiation therapy had to be redesigned in all 3 patients.

No significant differences were found in time elapsed between surgery and PRMR among patterns of enhancement growth (median time [range] between surgery and PRMR: "no growth" pattern, 35 days [26-45 days]; "focal growth," 36 days [28-42 days]; "global growth," 37 days [22-59 days]; "distant growth," 39 days [31-46 days]; $P=.827)$.

\section{DISCUSSION}

Our results suggest that performing an MR examination shortly before the start of treatment with radiation therapy may be highly relevant in the management of patients with glioblastoma who have undergone surgical resection. In this prospective study, some patients showed tumor growth before radiation therapy that required an enlargement of the target volume. In addition, performing a PRMR examination may reduce the number of pseudoprogression classifications by identifying a sample of patients in whom enhancement growth found in the first postradiation examination occurs before starting the treatment and not as a reaction to it. A third aspect of interest is that PRMR may provide independent prognostic information in patients with glioblastoma. In our study, the pattern of tumor growth on the PRMR examination satisfactorily correlated with patient survival, independent of the extent of surgical resection evaluated on the EPMR examination.

Glioblastoma is a very aggressive tumor with a high rate of proliferation and a dismal prognosis. MR imaging plays a very relevant role in the management of these tumors. An MR examination performed shortly after surgery, the EPMR, evaluates the extent of tumor resection and the presence of tumor remnant. This study is used to delineate the field for radiation therapy and will be the baseline examination to evaluate response to treatment. But unfortunately, glioblastoma may show relevant growth in short periods of time that could require enlarging the target volume of radiation therapy. ${ }^{9}$ In this respect, some studies suggest performing an MR examination shortly before treatment to validate the field of radiation therapy. ${ }^{9,13}$ However, to our knowledge, the impact of performing a PRMR examination has not been evaluated in depth, and current guidelines for glioblastoma management do not include performing PRMR. ${ }^{4,14}$ According to our findings, this examination seems to have a very positive impact on patient management. Four patients in our study showed relevant 
growth before radiation therapy that required enlarging the target volume.

Another relevant role of PRMR is related to the robustness of tumor follow-up. MR imaging plays a pivotal role in the detection of progressive disease in tumors that are not responding to treatment to provide the possibility of introducing an alternative treatment as soon as possible. Unfortunately, MR imaging assessments are not always totally accurate in detecting tumor progression. A paradigmatic example is the first postradiation MR examination, performed 2-6 weeks after treatment. In this examination, increased enhancement may be related to either true progression or pseudoprogression, and a reliable discrimination between these 2 entities cannot be performed by MR imaging alone. The pseudoprogression phenomenon has been considered to be induced by various nontumorous processes such as subacute radiation toxicity, treatment-related necrosis, and tissue inflammatory reaction. ${ }^{6,8}$ We believe that an additional possibility might be that the enlargement of the contrast-enhancing area occurs before starting radiation therapy and not as a consequence of treatment. Enlargement of the contrast-enhancing area before radiation therapy could be attributed to either tumor growth, surgical injury, or a combination of both. In any case, the demonstration that the contrast-enhancement enlargement has occurred before the initiation of radiation therapy would suggest that the concomitant treatment has been effective and that it has to be continued with adjuvant temozolamide. In our study, enhancement growth in the first postradiation MR examination was related to enhancement growth before treatment in 4 patients. The identification of these 4 patients improved the positive predictive value for true progression in the first postradiation MR examination from $56 \%$ to $75 \%$. This reduction in the number of cases classified as pseudoprogression would increase the robustness of the information that the radiologist brings to the multidisciplinary neuro-oncology unit to make the appropriate clinical decision when there is a precocious clinical deterioration of the patient.

Prognostic assessment of patients with glioblastoma is important by itself and may be relevant in deciding upon the best treatment to be offered in some particular cases. Some well-known prognostic factors are patient age, performance status, neurologic function, mental status, MGMT promoter methylation status, and extent of tumor resection. 5,11,12,15-22 The evolution of the tumor between EPMR and PRMR could provide information about proliferative behavior that might lead to additional predictive information about the outcome after therapy. Our study provides a positive argument for this possibility. We defined 4 patterns of tumor growth that satisfactorily correlated with patient survival. Showing no growth of the tumor was the most favorable option. In our study, patients with this pattern of growth survived longer (hazard ratio for death, 0.047) than patients showing a global growth of the enhancing tumor. An intermediate possibility was a focal pattern of growth (hazard ratio, 0.294).

Our study has several possible limitations. Distribution of the set of patients into 4 possibilities of tumor growth in the PRMR examination produced low numbers of patients in some groups. In particular, only 3 patients were included in the "distant growth" group. Although the survival of these patients was similar to the survival of patients with "global growth," a statistical anal- ysis could not be performed. Further studies could provide additional information about the clinical performance of this group of patients. Another consideration is the ranges of time elapsed between surgery and EPMR, between PRMR and radiation therapy, and between radiation therapy and follow-up with MR imaging. Shorter ranges of time could reduce the variability of the results and could provide more precise assessment of the parameters evaluated. However, all time ranges were within the standards considered in the diverse protocols, and time range did not influence the pattern of tumor growth found in the PRMR examination. A final concern is influence of a possible inflammatory component in the evolution found between EPMR and PRMR. To our knowledge, it is not possible to perform a precise analysis of the influence of an inflammatory component in the PRMR. We tried to avoid the uncertainty implicit in an association between a particular radiologic finding and a particular histologic situation by correlating our findings to patient survival and not to the presence or absence of tumor.

\section{CONCLUSIONS}

On the basis of our findings, we believe that performing an MR examination shortly before the start of radiation therapy would have a positive impact on the management of patients with glioblastoma by improving the planning of radiation therapy, reducing the ratio of pseudoprogression assessments, and providing additional predictive information. However, further multicenter studies in a larger set of patients are required to validate our findings.

Disclosures: Carles Majós-RELATED: Grant: Grupo Español de Investigación en Neurooncología (GEINO)* *Money paid to the institution.

\section{REFERENCES}

1. Ostrom QT, Gittleman H, Liao P, et al. CBTRUS statistical report: primary brain and central nervous system tumors diagnosed in the United States in 2007-2011. Neuro Oncol 2014;16(suppl 4):iv1-63 CrossRef Medline

2. Weller M, van den Bent M, Hopkins $K$, et al. EANO guideline for the diagnosis and treatment of anaplastic gliomas and glioblastoma. Lancet Oncol 2014;15:e395-403 CrossRef Medline

3. Stupp R, Mason WP, van den Bent MJ, et al. Radiotherapy plus concomitant and adjuvant temozolomide for glioblastoma. $N$ Engl J Med 2005;352:987-96 CrossRef Medline

4. NCCN Guidelines. NCCN guidelines for treatment of cancer by site. Central Nervous System Cancers. National Comprehensive Cancer Network. http://www.nccn.org/professionals/physician_gls/f_ guidelines.asp\#cns. Updated 2015. Accessed December 9, 2015

5. Albert FK, Forsting M, Sartor K, et al. Early postoperative magnetic resonance imaging after resection of malignant glioma: objective evaluation of residual tumor and its influence on regrowth and prognosis. Neurosurgery 1994;34:45-60; discussion 60-61 CrossRef Medline

6. Brandsma D, Stalpers L, Taal W, et al. Clinical features, mechanisms, and management of pseudoprogression in malignant gliomas. Lancet Oncol 2008;9:453-61 CrossRef Medline

7. Wen PY, Macdonald DR, Reardon DA, et al. Updated response assessment criteria for high-grade gliomas: response assessment in neuro-oncology working group. J Clin Oncol 2010;28:1963-72 CrossRef Medline

8. Hygino da Cruz LC Jr, Rodriguez I, Domingues RC, et al. Pseudoprogression and pseudoresponse: imaging challenges in the assess-

AJNR Am J Neuroradiol 37:2224-30 Dec 2016 www.ajnr.org 
ment of posttreatment glioma. AJNR Am J Neuroradiol 2011;32: 1978-85 CrossRef Medline

9. Pirzkall A, McGue C, Saraswathy S, et al. Tumor regrowth between surgery and initiation of adjuvant therapy in patients with newly diagnosed glioblastoma. Neuro Oncol 2009;11:842-52 CrossRef Medline

10. Valduvieco I, Verger E, Bruna J, et al. Impact of radiotherapy delay on survival in glioblastoma. Clin Transl Oncol 2013;15:272-82 CrossRef Medline

11. Ekinci G, Akpinar IN, Baltacioglu F, et al. Early-postoperative magnetic resonance imaging in glial tumors: prediction of tumor regrowth and recurrence. Eur J Radiol 2003;45:99-107 CrossRef Medline

12. Majós C, Cos M, Castañer S, et al. Early post-operative magnetic resonance imaging in glioblastoma: correlation among radiological findings and overall survival in 60 patients. Eur Radiol 2016;26: 1048-55 CrossRef Medline

13. Essig M, Anzalone N, Combs SE, et al. MR imaging of neoplastic central nervous system lesions: review and recommendations for current practice. AJNR Am J Neuroradiol 2012;33:803-17 CrossRef Medline

14. Stupp R, Tonn JC, Brada M, et al. High-grade malignant glioma: ESMO Clinical Practice Guidelines for diagnosis, treatment and follow-up. Ann Oncol 2010;21:90-93 CrossRef Medline

15. Gorlia T, van den Bent MJ, Hegi ME, et al. Nomograms for predicting survival of patients with newly diagnosed glioblastoma: prognostic factor analysis of EORTC and NCIC trial 26981-22981/CE.3. Lancet Oncol 2008;9:29-38 CrossRef Medline
16. Curran WJ Jr, Scott CB, Horton J, et al. Recursive partitioning analysis of prognostic factors in three Radiation Therapy Oncology Group malignant glioma trials. J Natl Cancer Inst 1993;85:704-10 CrossRef Medline

17. Scott CB, Scarantino C, Urtasun R, et al. Validation and predictive power of Radiation Therapy Oncology Group (RTOG) recursive partitioning analysis classes for malignant glioma patients: a report using RTOG90-06. Int J Radiat Oncol Biol Phys 1998;40:51-55 CrossRef Medline

18. Allahdini F, Amirjamshidi A, Reza-Zarei M, et al. Evaluating the prognostic factors effective on the outcome of patients with glioblastoma multiformis: does maximal resection of the tumor lengthen the median survival? World Neurosurg 2010;73:128-34 CrossRef Medline

19. McGirt MJ, Chaichana KL, Gathinji M, et al. Independent association of extent of resection with survival in patients with malignant brain astrocytoma. J Neurosurg 2009;110:156-62 CrossRef Medline

20. Lacroix M, Abi-Said D, Fourney DR, et al. A multivariate analysis of 416 patients with glioblastoma multiforme: prognosis, extent of resection, and survival. J Neurosurg 2001;95:190-98 CrossRef Medline

21. Sanai N, Berger MS. Glioma extent of resection and its impact on patient outcome. Neurosurgery 2008;62:753-64; discussion 264-66 CrossRef Medline

22. Smets T, Lawson TM, Grandin C, et al. Immediate post-operative MRI suggestive of the site and timing of glioblastoma recurrence after gross total resection: a retrospective longitudinal preliminary study. Eur Radiol 2013;23:1467-77 CrossRef Medline 